Mal J Nutr 26(3): 333-340, 2020

\title{
Validation and dimensional analysis of the eating behaviour pattern questionnaire among Malaysian university students
}

\author{
Maryam Kheirollahpour ${ }^{1}$, Asma Ahmad Shariff ${ }^{2,}{ }^{*}$, Amir Feisal Merican $^{3,4}$ \& \\ Mahmoud Danaee ${ }^{5^{*}}$
}

${ }^{1}$ Institute of Advanced Studies (IAS), University of Malaya, 50603 Kuala Lumpur, Malaysia; ${ }^{2}$ Mathematics Division, Centre For Foundation Studies in Science, University of Malaya, 50603 Kuala Lumpur, Malaysia; ${ }^{3}$ Institute of Biological Sciences, Faculty of Science, University of Malaya, Kuala Lumpur, Malaysia; ${ }^{4}$ Center of Research for Computational Sciences and Informatics in Biology, Bio industry, Environment, Agriculture and Healthcare (CRYSTAL), University of Malaya, 50603 Kuala Lumpur, Malaysia; ${ }^{5}$ Department of Social and Preventive Medicine, Faculty of Medicine, University of Malaya, 50603 Kuala Lumpur, Malaysia

\begin{abstract}
Introduction: Eating behaviour pattern is among the key behavioural factors that contribute to eating disorders. Hence, to evaluate the psychometric characteristics of the Eating Behaviour Pattern Questionnaire (EBPQ) that is used in epidemiological studies to measure the relationship between health outcomes and eating behaviour patterns, this study aimed to validate the adopted version of the EBPQ and to check the validity and reliability of this tool in University of Malaya, Malaysia. Methods: Exploratory factor analysis (EFA) was used to determine the most appropriate factor structure of EBPQ. Moreover, structural equation modelling (SEM) and confirmatory factor analysis (CFA) were applied to examine the convergent and discriminant validity of EBPQ. As for the participants of the study, multi-stage random sampling was used and 200 students (109 females and 91 males) from University of Malaya were chosen. Results: The EFA yielded nine components of EBPQ including emotional eating, eating outside, cultural habit, low-fat eating, meal skipping, snacking, healthy eating, planning for food and sweets, which explained $67.7 \%$ of the total variance. Furthermore, the Cronbach's $\alpha$ was about 0.8 for all components, which exhibited a high internal consistency among the obtained components. The results showed that the questionnaire had sufficient convergent and discriminant validity. Conclusion: The EBPQ was proven to be a reliable tool to measure the eating behaviour patterns in Malaysian university students. The presence of adequate validity and reliability supports this instrument's psychometric properties for future studies.
\end{abstract}

Keywords: Eating Behaviour Pattern Questionnaire, exploratory factor analysis, confirmatory factor analysis, structural equation modelling

\section{INTRODUCTION}

Recently, in both developed and developing countries, chronic diseases cause premature deaths and significant disabilities because of the changes in dietary patterns, eating behaviour,

\footnotetext{
*Corresponding author: Mahmoud Danaee Department of Social and Preventive Medicine, Faculty of Medicine, University of Malaya 50603 Kuala Lumpur, Malaysia.

Email: mdanaee@um.edu.my; Telephone: +60(3)-7967 4930

doi: https://doi.org/10.31246/mjn-2020-0005
} 
and lifestyle (Salekzamani, AsghariJafarabadi \& Dehghan, 2015). Behavioural factors such as eating behaviour pattern, is one of the most influential factors of weight gain and obesity (Chong et al., 2016). Therefore, modifying these main determinants of chronic diseases could decrease dietrelated diseases.

The Eating Behaviour Pattern Questionnaire (EBPQ) is used in epidemiological studies to measure the relationship between eating behaviour patterns and health outcomes, as well as to assess emotional, restrained and external eating behaviours (Van Strien et al., 1986; Cebolla et al., 2014; Dutton \& Dovey, 2016).

This questionnaire was adopted from previous studies with 51 items (Salekzamani et al., 2015; Schlundt et al., 2003). No study has yet examined the dimensions of EBPQ in context of Malaysian university students. Hence, this study aims to evaluate the dimensional structure of the adopted version of the EBPQ among Malaysian students, and to assess the instrument's reliability and validity.

\section{MATERIALS AND METHODS}

\section{Study design}

Structural equation modelling (SEM) is one of the complete and flexible techniques for testing and estimating the structural model of the overall relations among the dimensions of eating behaviour pattern questionnaire. In this study, the psychometric characteristics of EBPQ were checked through parallel analysis (PA) and exploratory factor analysis (EFA). EFA was applied to evaluate the structure and dimensions of the instrument, and confirmatory factor analysis (CFA) was used to assess the measurement model to test its convergent validity and construct reliability. Content validity was done through an expert panel review.

\section{Sample size and sampling method}

The participants were randomly selected from University of Malaya (semesters I and II, 2016 and 2017) through multi-stage random sampling technique (Cohen, 2007), with diverse socioeconomic status and without known physical or mental illnesses. A total of 17 faculties within University of Malaya was chosen. First, five faculties were randomly selected based on the highest percentage of students enrolled in each faculty. Second, the portion size and number of samples from different faculties were determined. Third, five departments were randomly chosen from each faculty and the number of classes for each semester was obtained from the administration office of each department. Fourth, the classes were randomly selected and finally the participants were randomly chosen among the local students. A package including the EBPQ, a consent form and information sheet were distributed among the participants. They were asked to complete the questionnaires individually and fill up a self-report demographic questionnaire about their age, educational level (Bachelor, Master, or Doctor of Philosophy degree), marital status (single or married), as well as their income. Other information regarding their weight and height were also selfreported.

The sufficient sample size for factor analysis and SEM was calculated using the power analysis method (Soper, 2015). Accordingly, the amount of $\beta$, $\alpha$, number of latent variables and the number of indicators were fixed. By considering $\beta=0.80$, number of latent variables $=9$, number of indicators $=51$ items and $\alpha=0.05$, the least number of sample calculated for partial least squares structural equation modelling (PLS-SEM) equaled to 200. 


\section{Ethical approval}

Ethical approval was obtained from the Faculty of Medicine, University of Malaya, [UM.TNC2/RC/H\&E/UMREC-63].

\section{Study instruments}

The original EBPQ used a 5-point Likert scale, from strongly disagree to strongly agree, to evaluate factors on eating behaviour patterns. It consisted of 51 items covering six factors: lowfat eating (11 items), snacking and convenience (10 items), emotional eating (8 items), planning ahead (6 items), meal skipping (7 items), and cultural-lifestyle behaviour (9 items). It also included a socio-demographic part encompassing information on age, gender, marital status, educational level and employment status. Statistical analysis was performed using the SPSS (ver. 23; Inc., Chicago, IL, USA) and Smart PLS (ver.3) was used for CFA analysis.

\section{Dimensional analysis}

Factor analysis was used to determine the correlation among the variables in a dataset by using Eigenvalues (Besnoy et al., 2016), which is frequently employed to argue for primary latent factors and/ or to validate questionnaires. To signify the number of factors/components, PA was used to reduce type I error as it gives an excessive number of factors. The PA suggested nine factors for the EBPQ, extracted through comparing the Eigenvalues of the actual data and the Eigenvalues of the simulated data (Çokluk \& Koçak, 2016).

\section{Exploratory factor analysis}

The Kaiser-Meyer-Olkin (KMO) value was over 0.82 for unobserved variables, signifying that the data were appropriate for factor analysis. Accordingly, EFA was performed through the principal axis factoring (PAF) extraction method and the Promax Rotation. Loading values above 0.4 were considered as satisfactory (Chong et al., 2016), while the number of factors (components) was identified based on the PA results.

\section{Reliability}

All statistical analyses were done at 95\% confidence level. Cronbach's $\alpha$ must be $>0.7$ and the item-total correlation should be $>0.4$ for each item. Cronbach's $\alpha$ was calculated to determine the scale's internal consistency for each dimension separately.

\section{Confirmatory factor analysis measurement model}

After establishing the components by EFA, the confirmatory factor analysis (CFA) was used to confirm each dimension and that the related items have sufficient construct validity through measurement model. In SEM analysis, measurement model is used to verify the convergent and discriminant validity. The measurement model deals with the relations between the latent (each component) and observed variables (related questions). It tests the reliability of the observed variables used to assess the latent variables. The CFA is used to assess the relationship between the indicators and associated latent variables. If the measurement model poorly fits the data, this means that some of the observed indicator variables are not reliable, thus preventing the researcher from proceeding to analyse the structural model. The items with low factor loadings are excluded from the measurement model. Moreover, when the fitness indices have reached the requirement level, the construct validity is achieved.

\section{Convergent validity}

Convergent and discriminant validity are the two main parts of CFA analysis. The convergent validity denotes the extent to which the indicators set can measure a construct. It is possible to evaluate the convergent validity at construct level through the average variance extracted. 
Therefore, composite reliability (CR) $>0.7$ is acceptable. The average variance extracted (AVE) should be $\geq 0.5$ (Hair $\mathrm{Jr}$ et al., 2016).

\section{Discriminant validity}

The discriminant validity reveals that each construct measurement should be different from other constructs. Therefore, for assessing the discriminant validity, the Fornell-Larcker criterion was used (Fornell \& Larcker, 1981).

\section{RESULTS}

Demographic characteristics are reported in Table 1. Exploratory factor analysis showed that a total of nine constructs were extracted from the data through PAF extraction method and the Promax Rotation method. The total variance for the EBPQ was $67.7 \%$. The first component or emotional eating with seven items included about $10.3 \%$ of the total variance. This percentage was followed by eating outside at about
9.0\% (six items), cultural habit (five items) at $7.0 \%$, low-fat eating (six items), meal skipping (five items), snacking (five items), healthy eating (five items), planning for food (five items), and sweets (four items).

Cronbach's $\alpha$ for all the components was satisfactory $(\alpha>0.8)$. The emotional eating sub-scale consisted of eight items $(\alpha=0.933)$. The next components were those with six items, including "lowfat eating" ( $\alpha=0.910)$, "cultural habit" $(\alpha=0.923)$ and "eating outside" $(\alpha=0.932)$. The rest were components with five items, including "healthy eating" $(\alpha=0.903)$, "snacking" ( $\alpha=0.908)$, "planning for food" $(\alpha=0.899)$ and "meal skipping" $(\alpha=0.937)$. The "sweets" component had four items $(\alpha=0.933)$.

SEM analysis showed that all the items based on the CFA were aligned to the established components by EFA. CFA analysis also revealed that all constructs (components) had sufficient internal consistency, convergent and

Table 1. Demographic characteristics of participants, $N=200$

\begin{tabular}{lc}
\hline Characteristics & Mean \pm SD \\
\hline Age (years) & $27.2 \pm 3.4$ \\
BMI $\left(\mathrm{kg} / \mathrm{m}^{2}\right)$ & Range $(22-36$ years) \\
Gender & $23.8 \pm 4.95$ \\
Range $(16.0-55.2)$ & \\
Male & \\
Female & $91(45.5)$ \\
Educational Level & $109(54.5)$ \\
Diploma & \\
Bachelor & $2(1.0)$ \\
Master & $134(67.0)$ \\
Doctor of Philosophy & $55(27.5)$ \\
Marital status & $9(4.5)$ \\
Single & \\
Married & $132(66.0)$ \\
Occupational status & $68(34.0)$ \\
Employed & \\
Unemployed & $18(4.0)$ \\
\hline
\end{tabular}


Table 2. Outer loading value and convergent validity for EBPQ

\begin{tabular}{|c|c|c|c|}
\hline Items & Outer Loading & $C R$ & $A V E$ \\
\hline Eating outside & & 0.939 & 0.719 \\
\hline EBP1 & 0.846 & & \\
\hline EBP13 & 0.832 & & \\
\hline EBP42 & 0.856 & & \\
\hline EBP43 & 0.875 & & \\
\hline EBP44 & 0.858 & & \\
\hline EBP50 & 0.820 & & \\
\hline Emotional eating & & 0.941 & 0.690 \\
\hline EBP2 & 0.854 & & \\
\hline EBP8 & 0.794 & & \\
\hline EBP9 & 0.849 & & \\
\hline EBP14 & 0.826 & & \\
\hline EBP19 & 0.828 & & \\
\hline EBP27 & 0.884 & & \\
\hline EBP32 & 0.776 & & \\
\hline Skipping meal & & 0.899 & 0.654 \\
\hline EBP17 & 0.897 & & \\
\hline EBP25 & 0.391 & & \\
\hline EBP36 & 0.881 & & \\
\hline EBP37 & 0.870 & & \\
\hline EBP48 & 0.882 & & \\
\hline Planning for food & & 0.812 & 0.465 \\
\hline EBP7 & 0.646 & & \\
\hline EBP20 & 0.641 & & \\
\hline EBP26 & 0.769 & & \\
\hline EBP35 & 0.637 & & \\
\hline EBP47 & 0.707 & & \\
\hline Snacking & & 0.931 & 0.731 \\
\hline EBP5 & 0.858 & & \\
\hline EBP10 & 0.882 & & \\
\hline EBP16 & 0.848 & & \\
\hline EBP2 1 & 0.877 & & \\
\hline EBP41 & 0.809 & & \\
\hline Low-fat eating & & 0.931 & 0.691 \\
\hline EBP3 & 0.781 & & \\
\hline EBP4 & 0.807 & & \\
\hline EBP11 & 0.835 & & \\
\hline EBP29 & 0.890 & & \\
\hline EBP39 & 0.857 & & \\
\hline EBP49 & 0.814 & & \\
\hline Sweets & & 0.953 & 0.834 \\
\hline EBP12 & 0.926 & & \\
\hline EBP30 & 0.925 & & \\
\hline EBP40 & 0.898 & & \\
\hline EBP46 & 0.903 & & \\
\hline Healthy eating & & 0.928 & 0.721 \\
\hline EBP6 & 0.850 & & \\
\hline EBP18 & 0.900 & & \\
\hline EBP22 & 0.846 & & \\
\hline EBP24 & 0.848 & & \\
\hline EBP45 & 0.798 & & \\
\hline
\end{tabular}


discriminant validity.

Indeed, the factor loading results supported the results of the factor analysis. All outer loadings were $>0.700$. However, item 38 (from low-fat eating), item 8 (from emotional eating) and item 51 were removed as their loading values were $<0.400$ (Table 2). The indicator reliability was assessed by outer loadings, yet, Cronbach's $\alpha$ is the conventional criterion for internal consistency. Results indicated that both criteria (CR and Cronbach's $\alpha$ ) were satisfactory and that the instrument had sufficient internal consistency.

According to Table 2, CR was in the range of 0.812 to 0.933 . CR was introduced to measure internal consistency reliability. AVE was $>0.650$ for all constructs, except for planning for food. However, if the AVE value was not satisfactory, the researcher may decide to keep or remove that particular construct. In this case, if the CR was $>0.7$, then that construct may be retained (Hair Jr et al., 2016). The convergent validity was assessed through satisfactory level of $\mathrm{CR}$ and AVE. Therefore, each set of specific questions could only measure the specific component (i.e., four questions specifically could measure the sweet component).

The discriminant validity was assessed. According to the results of Fornell-Larcker method for each construct, the AVE was more than every squared correlation between the constructs (Table 3). Consequently, all constructs in the measurement model, which were based on the questionnaire (EBPQ), had sufficient discriminant validity, which meant that each component measured different concepts.

\section{DISCUSSION}

The current study enjoyed novelty in terms of presenting the adopted version of the EBPQ among Malaysian students. The strong point of this study was using advanced methods of analysis such as parallel analysis for psychometric analysis, power analysis to apply adequate sample size and SEM analysis.

The components of EBPQ were determined, and the validity and reliability of EBPQ were checked. Using the exploratory factor analysis, components of EBPQ were extracted including items describing eating behaviour patterns that were related to unhealthy and healthy eating behaviours. The final EBPQ was reconstructed with 48 items. Three items including items 8,38 and 51 were removed due to factor loadings $<0.400$. These findings were in line with

Table 3. Discriminant validity

\begin{tabular}{lccccccccc}
\hline$E B P Q$ & $E M$ & $H E$ & $E O$ & $S$ & $M S$ & $S N$ & $L F$ & $P L$ & $C H$ \\
\hline Emotional eating & 0.685 & & & & & & & & \\
Healthy eating & 0.019 & 0.806 & & & & & & & \\
Eating outside & 0.229 & -0.123 & 0.729 & & & & & & \\
Sweets & 0.429 & 0.166 & 0.299 & 0.754 & & & & & \\
Meal skipping & 0.250 & 0.311 & 0.105 & 0.359 & 0.756 & & & & \\
Snacking & 0.594 & -0.035 & 0.374 & 0.361 & 0.222 & 0.768 & & & \\
Low-fat eating & 0.053 & 0.141 & 0.066 & -0.017 & 0.074 & 0.129 & 0.687 & & \\
Planning food & -0.043 & 0.150 & 0.001 & 0.193 & 0.101 & -0.017 & 0.383 & 0.662 & \\
Cultural habits & 0.271 & 0.031 & 0.227 & 0.277 & 0.172 & 0.241 & -0.016 & 0.140 & 0.698 \\
\hline
\end{tabular}

EM: emotional eating; HE: healthy eating; EO: eating out; SB: sweets; MS: meal skipping; SN: snacking; LF: low-fat eating; PL: planning food; $\mathrm{CH}$ : cultural habits 
the original factor structure (Van Strien et al., 1986). Similarly, in another study, these three items were also removed from further analysis due to issues with factor structure (Cebolla et al., 2014). The original EBPQ was established with six factors (Schlundt 2003).

Similarly, the "low-fat eating" subscale was split into healthy eating and low-fat eating (Kee et al., 2008). The "snacking and convenience" factor was split into three sub-scales - snacking, eating out, as well as sweets. Therefore, the nine patterns of eating behaviours identified were (1) emotional eating, (2) eating outside, (3) cultural habits, (4) low-fat eating, (5) meal skipping, (6) snacking, (7) healthy eating, (8) planning for food and (9) sweets. Cronbach's $\alpha$ revealed a high internal consistency among the items of each component. Independent factors in the EBPQ denoted that multiple dimensions characterised youth eating behaviours, while previous studies have used the EBPQ to show a unitary construct (Goldbacher et al., 2012). It might be concluded that the EBPQ does not replace traditional dietary assessment methods. Instead, it is a measurement of eating patterns that is possibly pertinent to disease prevention and health outcomes.

Another aim of this study was to assess the internal structure of the EBPQ and to evaluate the instrument's validity and reliability. The result showed that the EBPQ has adequate psychometric characteristics and can be used in clinical practice to better understand eating behaviour patterns. Moreover, the discriminant validity was assessed and the results indicated that the ninefactor EBPQ has adequate discriminant validity. Therefore, the individual differences in choosing healthy or unhealthy eating behaviour pattern may not reflect anything more than a general eating behaviour pattern. The results of discriminant validity also showed that each unique dimension of EBPQ was distinct from one another. The findings were consistent with those of a similar nature (Salekzamani et al., 2015). The findings verified that the adopted version of the EBPQ had the theoretical factor structure. Furthermore, previous evaluations of the EBPQ's factor structure were limited to the samples of normal weight children and binge-eating women with low diversity (Schlundt et al., 2003). This research presented good information about the factor structure in a more heterogeneous sample.

\section{CONCLUSION}

The EBPQ was a suitable tool for measuring the eating behaviour pattern of the participants and it was consistent throughout. However, the exclusion criteria (e.g., the absence of significant medical conditions and the ability to be physically active) limited the possibility of generalising these findings to the general public. Thus, future direction for similar studies is to apply EBPQ in larger sample sizes containing all categories of the society. The findings could be used for further statistical and epidemiological research to understand the psychometric characteristics of research instruments.

\section{Acknowledgement}

Not applicable.

\section{Authors' contribution}

MK, involved in sample collection, data analysis, computational analyses, result interpretation, manuscript drafting and revision; AAS, designed and supervised the study, collected the samples, drafted, revised and critically reviewed the manuscript; AFM, designed and supervised the study and critically reviewed the manuscript; MD, performed computational analyses and involved in data analysis, result interpretation, and manuscript revision, designed and supervised the study and critically reviewed the manuscript. All authors have final approval on the manuscript to be published.

\section{Conflict of interest}

This manuscript has not been published elsewhere and is not under consideration by other journals. All authors have approved the manuscript and agree with submission to the Malaysian Journal 
of Nutrition. We declare that there is no conflict of interest regarding the publication of this study.

\section{References}

Besnoy KD, Dantzler J, Besnoy LR \& Byrne C (2016). Using Exploratory and Confirmatory Factor Analysis to Measure Construct Validity of the Traits, Aptitudes, and Behaviors Scale (TABS). J Educ Gift 39(1):3-22.

Cebolla A, Barrada J, Van Strien T, Oliver E \& Baños R (2014). Validation of the Dutch Eating Behavior Questionnaire (DEBQ) in a sample of Spanish women. Appetite 73:58-64.

Chong, M.F.F., Ayob, M.N.I.M., Chong, K.J., Tai, E.S., Khoo, C.M., Leow, M.K.S., Lee, Y.S., Tham, K.W., Venkataraman, K., Meaney, M.J. and Wee, H.L (2016). Psychometric analysis of an eating behaviour questionnaire for an overweight and obese Chinese population in Singapore. Appetite 101:119-124.

Cohen, L., Manion, L., \& Morrison, K, (2007). Research methods in education. Taylor and Francis Group, Routledge, London and Newyork.

Çoklu Ö \& Koçak D (2016). Using Horn's Parallel Analysis Method in Exploratory Factor Analysis for Determining the Number of Factors. Educational Sciences: Theory \& Practice 16(2):537-551.

Dutton E and Dovey TM (2016). Validation of the Dutch Eating Behaviour Questionnaire (DEBQ) among Maltese women. Appetite: 107:9-14.

Fornell C \& Larcker DF (1981). Evaluating structural equation models with unobservable variables and measurement error. J Mark Res $18(1): 39-50$.
Goldbacher E, Grunwald H, LaGrotte C, Klotz A, Oliver T, Musliner K \& Foster G (2012). Factor structure of the Emotional Eating Scale in overweight and obese adults seeking treatment. Appetite 59(2):610-615.

Hair Jr JF, Hult GTM, Ringle C \& Sarstedt M (2016). A primer on partial least squares structural equation modeling (PLS-SEM) (pp. 105-109). Sage Publications, California.

Kee CC, Jamaiyah H, Safiza MN, Geeta A, Khor GL, Suzana S, Jamalludin AR, Rahmah R, Ahmad AZ \& Ruzita AT (2008). Abdominal Obesity in Malaysian Adults: National Health and Morbidity Survey III (NHMS III, 2006). Mal $J$ Nutr 14(2):125-135.

Salekzamani S, Asghari-Jafarabadi M \& Dehghan $P$ (2015). Validity, Reliability and Feasibility of the Eating Behavior Pattern Questionnaire (EBPQ) among Iranian female students. Health Health Promot Perspect 5(2): 128.

Schlundt DG, Hargreaves MK \& Buchowski MS (2003). The eating behavior patterns questionnaire predicts dietary fat intake in African American women. $J$ Am Diet Assoc 103(3):338-345.

Soper DS (2015). A-priori sample size calculator for structural equation models. From http:// www. danielsoper. com/statcalc [Retrieved December 12 2015] [Software].

Van Strien T, Frijters JE, Bergers G \& Defares PB (1986). The Dutch Eating Behavior Questionnaire (DEBQ) for assessment of restrained, emotional, and external eating behavior. J Eat Disord 5(2):295-315. 